\title{
Morphology-independent background estimation for extended gamma-ray sources
}

\author{
Ruo-Yu Shang ${ }^{a, *}$ and Vladimir V. Vassiliev ${ }^{a}$ \\ ${ }^{a}$ University of California, Los Angeles, Department of Physics and Astronomy, Los Angeles, U.S.A. \\ E-mail: rshang@astro.ucla.edu, vvv@astro.ucla.edu
}

Detection of the gamma-ray sources with the angular extend of the order of one degree or larger represents a significant challenge for the imaging Cherenkov atmospheric telescopes (IACTs) due to the lack of reliable estimation of the background cosmic rays (CRs) in the telescope field of view of a few degrees. We present a novel method, which allows estimation of the CR background without the prior assumption about the morphology of the gamma-ray emission from a putative source with large angular extension. This novel method, morphology-independent background estimation (MIBE), utilises the "cosmic-ray-like" events (those that fail gamma-hadron-separation cuts based on shower-shape parameters) collected from the given field to estimate the " $\gamma$-raylike" background in the same field. The method is shown to reduce the systematic error of the background estimate comparing to the conventional approaches for the analysis of gammaray emission from sources with large angular extension and with unknown morphology. This submission explains the methodology and presents the validation of the MIBE using the gammaray-free data obtained by the VERITAS (Very Energetic Radiation Imaging Telescope Array System).

$37^{\text {th }}$ International Cosmic Ray Conference (ICRC 2021)

July 12th-23rd, 2021

Online - Berlin, Germany

\footnotetext{
*Presenter
} 


\section{Introduction}

Very-high-energy (VHE) $\gamma$-ray astronomy probes the Universe in the energy range from about $30 \mathrm{MeV}$ [Fermi-Large Area Telescope (LAT), $30 \mathrm{MeV}-300 \mathrm{GeV}$ ] to above $1 \mathrm{PeV}$ [High Altitude Water Cherenkov (HAWC) experiment and Large High Altitude Air Shower Observatory (LHAASO), $300 \mathrm{GeV} \mathrm{TeV}-1000 \mathrm{TeV}$ ]. Space-based Fermi-LAT and ground-based HAWC and LHAASO are survey observatories which have instantaneous field of view exceeding 2 steradians. The detailed astrophysical studies of the $\gamma$-ray sources in the energy range between $100 \mathrm{GeV}$ and $10 \mathrm{TeV}$ are benefited from the observations conducted by the ground-based arrays of Imaging atmospheric Cherenkov telescopes (IACTs), such as H.E.S.S., MAGIC and VERITAS. IACT arrays are characterized by very large photon collecting areas in access of several hundred thousand square meters, excellent angular and energy resolutions but they are limited by the relatively small instantaneous field of view (FoV) of $<5$ degrees.

To date, IACTs detected and studied more than a hundred of $\gamma$-ray sources. Vast majority of them are point sources with the angular extend comparable or smaller than the $\gamma$-ray point spread function (PSF) of IACTs of a few tens of a degree. Only a relatively small fraction of sources detected and explored by IACTs exhibit angular extensions much larger than their PSF and and only a few have angular size larger than 1 degree or comparable to the IACT FoV. Contrary, the fraction of large angular extension mostly galactic sources in Fermi-LAT and HAWC catalogs is significant, which points to the superior ability of these instruments to detect such sources and simultaneously shows relatively low sensitivity of IACT arrays in finding and studying them. The research of morphology resolved spectroscopy of such sources is very valuable for VHE astrophysics as it provides testing grounds for theories of particle acceleration and interaction with the interstellar medium (ISM) as well as supernova remnant (SNR) and pulsar wind nebula (PWN) evolution [8] [4].

The limitation in detecting large angular extend $\gamma$-ray sources utilizing imaging atmospheric Cherenkov technique arises from the deficiency in estimation of the cosmic ray (CR) background within the relatively small FoV of IACTs. Normally, an assumption about the morphology of the putative source is needed to isolate $\gamma$-ray free regions in the IACT observatory FoV for $\gamma$-ray-like $\mathrm{CR}$ background estimation and it becomes unreliable for sources with large angular extension. The alternative conventional methods for deriving $\gamma$-ray-like CR background from other observational fields suffer from the significant systematic errors, which ultimately limit the sensitivity [2]. Motivated by the need to increase the sensitivity of IACT arrays for detecting astrophysical sources with large angular extension, this paper introduces a novel morphology-independent background estimation (MIBE) method developed for the analyses of IACT data, which does not require morphology assumptions and can estimate $\gamma$-ray-like CR background from the same FoV of source observations.

\section{Gamma v.s. hadron discrimination parameters}

The transverse development of atmospheric cascades (showers) initiated by a cosmic-ray is distinguishable from that of initiated by a $\gamma$-ray due to the significant transverse momentum provided by massive pion decays. The transverse and longitudinal developments of the shower are 
characterized as an ellipse in the Hillas parametrization [5] [3]. The length and the width of a shower depends on the primary particle energy $E$ and the shower impact parameter $R$ (the distance from the telescope to the shower axis). In order to minimize the dependency of the background discrimination parameters on these factors, the length and the width of a shower image are compared against a set of $\gamma$-ray shower simulations at a given interval of shower size $s$ (the total charge in all pixels of the shower image recorded by IACT) and impact parameter $R$ [1] [7] [6]. The derived quantities (known as shower shape background discrimination parameters) are the mean reduced scaled length $(M S C L)$

$$
M S C L=\frac{1}{N} \sum_{i=1}^{N} \frac{l_{\mathbf{o b s}, i}(s, R)-\bar{l}_{\mathbf{s i m}}(s, R)}{\sigma_{l, \operatorname{sim}}(s, R)},
$$

and the mean reduced scaled width $(M S C W)$

$$
M S C W=\frac{1}{N} \sum_{i=1}^{N} \frac{w_{\mathbf{o b s}, i}(s, R)-\bar{w}_{\mathbf{s i m}}(s, R)}{\sigma_{w, \operatorname{sim}}(s, R)},
$$

where $\mathrm{N}$ is the number of telescopes, $l_{\mathbf{o b s}, i}\left(w_{\mathbf{o b s}, i}\right)$ is the shower length (width) observed by the $i$ th telescope, $\bar{l}_{\text {sim }}\left(\bar{w}_{\text {sim }}\right)$ is the expected length (width) of the simulated $\gamma$-rays, and $\sigma_{l \text {,sim }}\left(\sigma_{w}\right.$,sim $)$ is the $90 \%$ containment variation of the length and width of the simulated events.

In this paper, we refer to the events passing the background discrimination shower shape cuts defined on the two dimensional (MSCL, MSCW) background distribution map as the $\gamma$-ray-like events, and to the events failing these cuts as the cosmic-ray-like events.

\section{Low-rank modeling of the (MSCL, MSCW) matrix of cosmic-ray background distribution}

The novel MIBE method is based on an empirical observation of the properties of cosmicray event distribution in the two dimensional space of the shower shape parameters, $M S C W$ and $M S C L$. The distribution is described by a matrix whose column and row indices label $M S C W$ and $M S C L$ bins of the shower events, respectively, and the elements of the matrix show the numbers of events in the bins. An example of such a matrix $M^{\mathrm{ON}}$ is shown in Figure 1 (a), in which the events are assembled from the VERITAS observations data of Segue 1 dwarf galaxy. The choice of the boundaries of the matrix is $(-0.6,1.8)$ in $M S C L$ and $(-0.6,1.6)$ in $M S C W$, and the $\gamma$-ray-like background region for the $\gamma$-ray candidate events is defined by the red box, the region outside the $\gamma$-ray region is defined as the cosmic-ray-like region. A straightforward method to initially estimate the cosmic-ray background in the $\gamma$-ray region of $M^{\mathrm{ON}}$ is to build a template matrix $M^{\mathrm{OFF}}$ by collecting events from $\gamma$-ray-free fields (excluding Segue 1) with similar telescope pointing elevations, trigger rates and the night-sky-brightness level as is in the Segue 1 observations and properly normalizing it to match total number of events in "ON" and "OFF" matrices. The example of template matrix is illustrated in Figure 1 (b). The residual matrix, defined as $M^{\mathrm{ON}}-M^{\mathrm{OFF}}$ and shown in Figure 1 (c), reveals the mis-modeling of $M^{\mathrm{OFF}}$ as it shows underestimation of the CR background in the $\gamma$-ray-like region.

It has been empirically observed that the CR background distribution described by $M^{\text {OFF }}$ matrices for different observing fields, although could be significantly different, they all admit 
compact singular-value decomposition (SVD) in which first three largest singular values describe the total $\gamma$-ray-like background with an accuracy approaching $1 \%$. It is this property, which motivates us to improve initial $M^{\mathrm{OFF}}$ estimate by applying SVD with the goal to correct the mis-modeling of the residual matrix based on the necessity of compact SVD description in the cosmic-ray-like region of matrix $M$.

To illustrate this, the singular values of the matrices of multiple signal-free fields in different energy ranges are shown in Figure 2. The primary singular values of the matrices are defined as the singular values whose sizes are larger than the singular value at the "elbow" position of the curves in Figure 2. The number of primary singular values is $\leq 3$ and declines as the energy of shower events increases. In the low energy range $\left(E<10^{2.66} \mathrm{GeV}\right)$, three primary singular values are needed to describe the matrices; in the high energy range $\left(E>10^{3.0} \mathrm{GeV}\right)$, only one primary singular value is required. Inspired by this empirical finding we recover the cosmic-ray background in the $\gamma$-ray region of the matrix in a given field of interest, through the perturbation of properly matched initial $M^{\mathrm{OFF}}$ matrix using compact SVD and regularization attempting to force reduction of its rank. Implicitly, this method exploits the underling complex correlations between cosmic-ray-like regions and $\gamma$-ray-like regions of the $M$ matrices populated exclusively by cosmic rays.

Suppose that the SVD of the reconstructed cosmic-ray background matrix of a field of interest (ON field) is

$$
\tilde{M}^{\mathrm{ON}}=U \Sigma V^{\dagger}=\sum_{k} \sigma_{k}^{\mathrm{ON}} \vec{u}_{k}^{\mathrm{ON}} \vec{v}_{k}^{\mathrm{ON}}
$$

where

$$
\begin{gathered}
\Sigma=\left[\begin{array}{ccc}
\sigma_{1}^{\mathrm{ON}} & 0 & \\
0 & \sigma_{2}^{\mathrm{ON}} & \\
& & \ddots
\end{array}\right], \\
U=\left[\begin{array}{lll}
\vec{u}_{1}^{\mathrm{ON}} & \vec{u}_{2}^{\mathrm{ON}} & \cdots
\end{array}\right], V=\left[\begin{array}{lll}
\vec{v}_{1}^{\mathrm{ON}} & \vec{v}_{2}^{\mathrm{ON}} & \cdots
\end{array}\right],
\end{gathered}
$$

and $\sigma_{k}^{\mathrm{ON}}$ is the $k$-th largest singular value of $M^{\mathrm{ON}}$ that is shown in Figure 2. A perturbation theory is constructed as $\sigma_{k}^{\mathrm{ON}}=\sigma_{k}^{\mathrm{OFF}}+\delta \sigma_{k}, \vec{u}_{k}^{\mathrm{ON}}=\vec{u}_{k}^{\mathrm{OFF}}+\delta \vec{u}_{k}$, and $\vec{v}_{k}^{\mathrm{ON}}=\vec{v}_{k}^{\mathrm{OFF}}+\delta \vec{v}_{k}$. The initial condition $\sigma_{k}^{\mathrm{OFF}}, \vec{u}_{k}^{\mathrm{OFF}}$, and $\vec{v}_{k}^{\mathrm{OFF}}$ are constructed from properly matched OFF fields ( $M^{\mathrm{OFF}}$ ). The perturbations, $\delta \sigma_{k}, \delta \vec{u}_{k}$ and $\delta \vec{v}_{k}$, are determined by minimizing the Frobenius norm of the difference matrix $M^{\mathrm{ON}}-\tilde{M}^{\mathrm{ON}}$ in the cosmic-ray-like region and requiring the regularization on the perturbations, i.e.

$$
\text { Min. }\left\|M^{\mathrm{ON}}-\tilde{M}^{\mathrm{ON}}\right\|_{\text {blind }}^{2} \text { subject to } R\left(\delta \sigma_{k}, \delta \vec{u}_{k}, \delta \vec{v}_{k}\right)=0 \text {, }
$$

where $M^{\mathrm{ON}}$ is the observed matrix of the ON field, and $\tilde{M}^{\mathrm{ON}}$ is the reconstructed ON field matrix. The regularization includes truncating the perturbations of singular values by only allowing the perturbations to the primary singular values, i.e. $\delta \sigma_{k}=0$ for $k \notin$ primary indices, and imposing correlations among the perturbation parameters.

The result of the Frobenius norm of $M$ minimization (with $\gamma$-ray-like region excluded) and the low-rank regularization is that the systematic error of the background prediction is significantly reduced in low energy region $(<500 \mathrm{GeV})$. An example of the perturbation solution is shown in Figure $1(\mathrm{~d})$, where one can see that the discrepancies in both cosmic-ray-like region and $\gamma$-ray 
region are reduced in comparison with that of the initial $M^{\mathrm{OFF}}$ template. In order to evaluation the performance of the MIBE method, a "figure of merit" metric is defined as

$$
\epsilon^{\text {Perturb }}=\frac{\left|N_{\gamma \text { bkg }}-N_{\text {model }}^{\text {Perturb }}\right|}{N_{\gamma \mathrm{bkg}}},
$$

where $N_{\gamma \text { bkg }}$ is the observed background count in the $\gamma$-ray-like region from a signal-free field, and $N_{\text {model }}^{\text {Perturb }}$ is the predicted background count in the $\gamma$-ray-like region using the model of perturbation. The averaged $\epsilon^{\text {Perturb }}$ from multiple signal-free fields represents the relative systematic error on the background estimation of the perturbation solution. The perturbation method couples the variation in the $\gamma$-ray-like region to the variation in the cosmic-ray-like region, so that one can use the cosmic-ray-like events, which are collected at the same time as the $\gamma$-ray-like events and contain the information of the ON field observation conditions, to minimize the systematic uncertainty on background normalization. Table 1 summarizes the measured relative systematic errors at different shower energy ranges.

\begin{tabular}{|c|c|c|}
\hline energy range & $\left\langle\epsilon^{\text {OFF }}\right\rangle$ & $\left\langle\epsilon^{\text {Perturb }}>\right.$ \\
\hline$\left[10^{2.0}, 10^{2.33}\right] \mathrm{GeV}$ & $2.8 \%$ & $1.2 \%$ \\
{$\left[10^{2.33}, 10^{2.66}\right] \mathrm{GeV}$} & $3.5 \%$ & $1.1 \%$ \\
{$\left[10^{2.66}, 10^{3.0}\right] \mathrm{GeV}$} & $3.3 \%$ & $2.5 \%$ \\
{$\left[10^{3.0}, 10^{3.33}\right] \mathrm{GeV}$} & $3.8 \%$ & $3.8 \%$ \\
{$\left[10^{3.33}, 10^{3.66}\right] \mathrm{GeV}$} & $6.9 \%$ & $6.1 \%$ \\
$>10^{3.66} \mathrm{GeV}$ & $24 \%$ & $24 \%$ \\
\hline
\end{tabular}

Table 1: The measured systematic errors, $<\epsilon^{\mathrm{OFF}}>$ (initial template estimation) and $<\epsilon^{\text {Perturb }}>$ (perturbation estimation), at different shower energy ranges. The measurement of the systematic errors uses 17 extragalactic point source observations with the point sources masked with a radius of $0.3^{\circ}$.

\section{MIBE application example}

The morphology-independent background estimation obtained as perturbation solution allows the detection of genuine $\gamma$-ray source morphology, which is free from the bias due to the conventional morphology-dependent-exclusion-regions estimation of the $\gamma$-ray-like background. To detect the morphology-dependent spectroscopic properties of a source, a $\gamma$-ray-like background probability template in the camera coordinate $\vec{\theta}$ is built as

$$
P(E, \vec{\theta})=\frac{w(E, \vec{\theta}) N_{\mathrm{CR}}(E, \vec{\theta})}{\sum_{\vec{\theta}} w(E, \vec{\theta}) N_{\mathrm{CR}}(E, \vec{\theta})},
$$

where $E$ is the shower energy, and $N_{\mathrm{CR}}(E, \vec{\theta})$ is the distribution of cosmic-ray-like events sampled from the ON field. The $w(E, \vec{\theta})$ factor, which is measured as

$$
w(E, \vec{\theta})=\frac{N_{\gamma \mathrm{bkg}}^{\mathrm{OFF}}(E, \vec{\theta})}{N_{\mathrm{CR}}^{\mathrm{OFF}}(E, \vec{\theta})},
$$




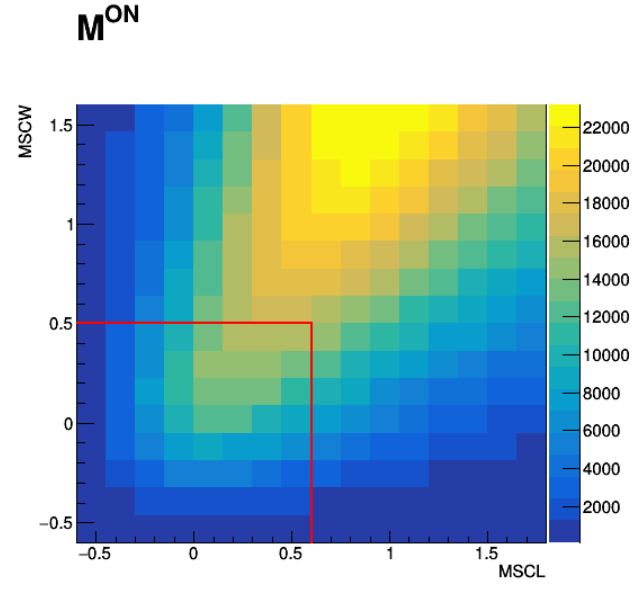

(a)

$$
M^{\text {ON }}-M^{\text {OFF }}
$$

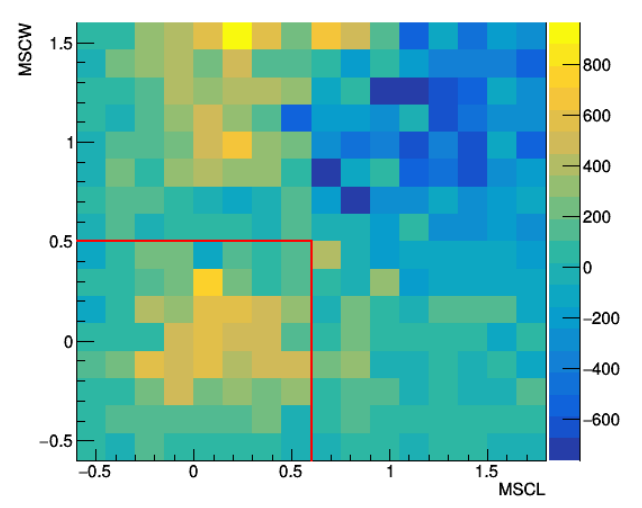

(c)

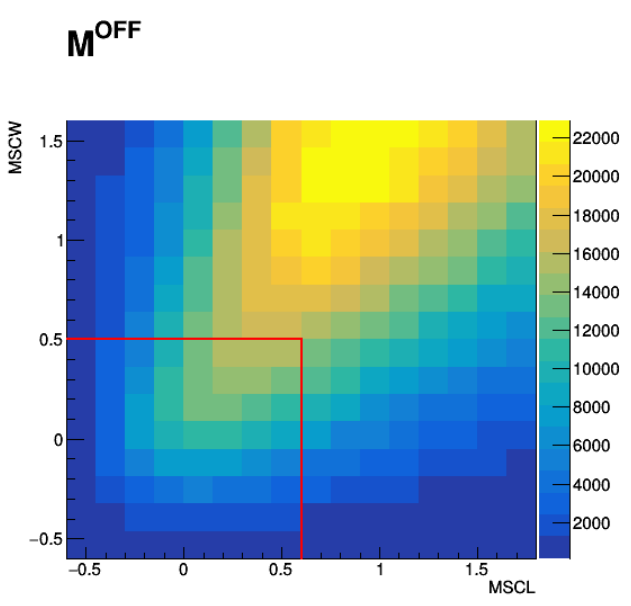

(b)

$$
M^{\mathrm{ON}}-\tilde{M}^{\mathrm{ON}}
$$

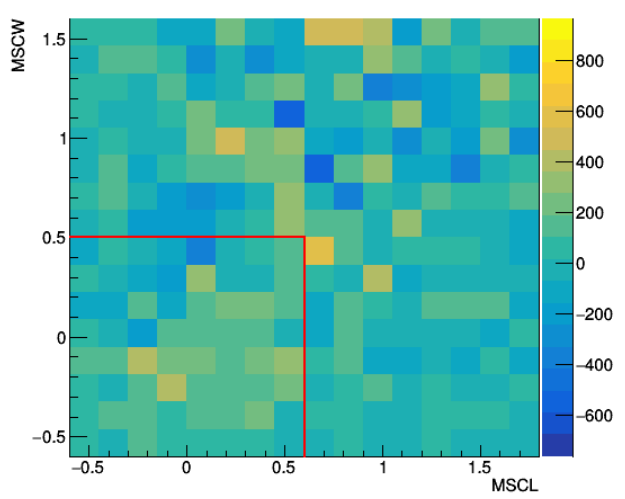

(d)

Figure 1: The cosmic-ray event distribution as a matrix in $M S C L-M S C W$ space. (a) ON field matrix $M^{\mathrm{ON}}$, the events are sampled from a $\gamma$-ray-free dark field of Segue 1 in VERITAS data by masking the point-like extragalactic source with a cut of angular radius of $0.45^{\circ}$. The events with reconstructed arrival directions falling outside of a radius of $2^{\circ}$ around the center of the camera coordinate are rejected. The red lines define the $\gamma$-ray-like region, the region outside the red box is defined as the cosmic-ray-like region. (b) OFF field matrix $M^{\mathrm{OFF}}$, the events are sampled from several $\gamma$-ray-free dark fields (excluding Segue 1) in VERITAS data by masking the point-like extragalactic sources. (c) The difference matrix $M^{\mathrm{ON}}-M^{\mathrm{OFF}}$. (d) The difference matrix $M^{\mathrm{ON}}-\tilde{M}^{\mathrm{ON}}$. 


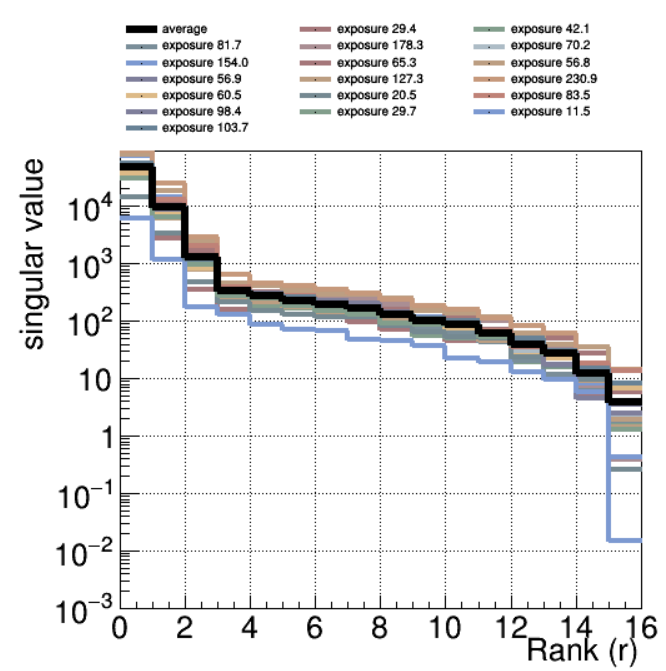

(a)

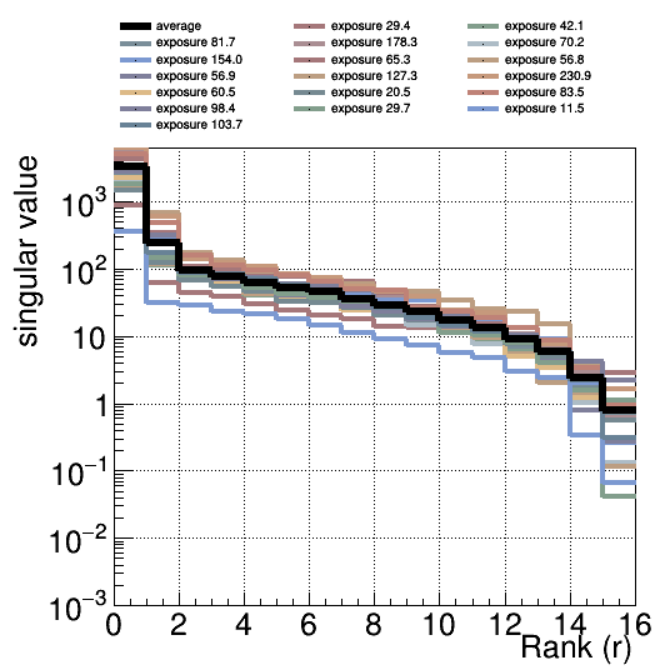

(b)

Figure 2: The singular values of the shower shape parameter matrices sampled from signal-free field VERITAS data in shower event energy range $E \in\left[10^{2.33}, 10^{2.66}\right] \mathrm{GeV}$ (a) and $E \in\left[10^{3.0}, 10^{3.33}\right] \mathrm{GeV}$ (b), respectively. The number of the primary singular values is defined by the "elbow" position of the curve, which is $r=3$ in $E \in\left[10^{2.0}, 10^{2.66}\right] \mathrm{GeV}$ range, $r=2$ in $E \in\left[10^{2.66}, 10^{3.33}\right] \mathrm{GeV}$ range, and $r=1$ in $E>10^{3.33} \mathrm{GeV}$ range. The legend shows the exposure hours of each signal-free field observation.

is a correction factor to account for the acceptance difference as function of shower energy and location in camera coordinate between $\gamma$-like events and cosmic-ray-like events measured from a set of OFF fields. This template $P(E, \vec{\theta})$ is then normalized by the MIBE perturbation solution

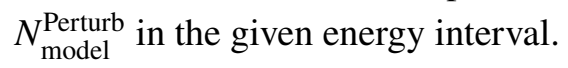

The measurements of the morphology resolved spectroscopic properties of the $\gamma$-ray sources with large angular extension provide opportunities to study the acceleration of VHE particles, their transport through medium and their energy losses, and, hence, contribute to the study of the evolution of pulsars and SNRs. An example of the morphology-dependent measurement of spectral-indexes is shown in Figure 3. In this illustration, a signal-free VERITAS observation is injected with simulated $\gamma$-ray events. The simulated $\gamma$-ray sources are extended (Gaussian profile with width of $0.5^{\circ}$ ) and have different spectral indices, 2.5 and 3.5. The skymap of the spectral index is shown in Figure 3, in which reveals the correctly reconstructed spectral indices of the two sources.

\section{Conclusion}

The MIBE method described in this paper has been developed for the analysis of IACT observation of VHE $\gamma$-ray sources with large angular extend, which can be comparable to the FoV of the observatory. The MIBE offers significant reduction of the systematic errors in $\gamma$-ray-like background estimation thereby increasing the sensitivity of IACT arrays for the detection of such sources. The ability of the method to estimate background from the same observation field enables 


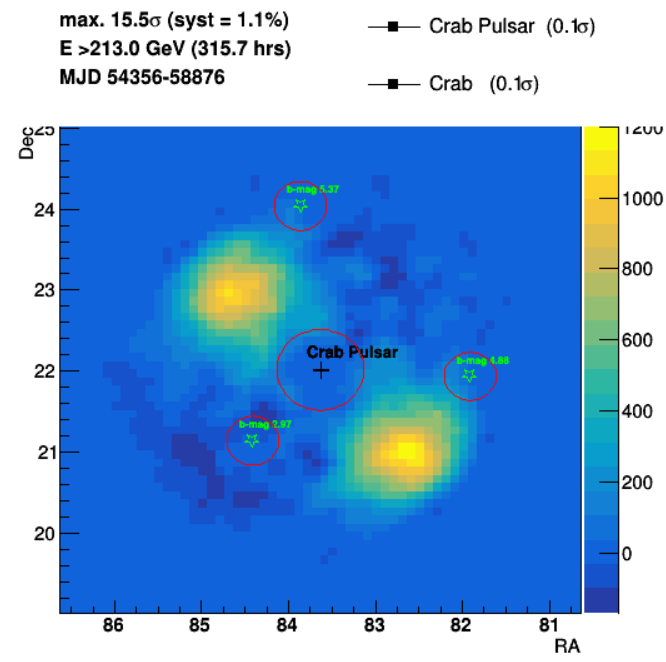

(a)

\section{E $>213.0 \mathrm{GeV}(315.7 \mathrm{hrs})$ \\ MJD 54356-58876}

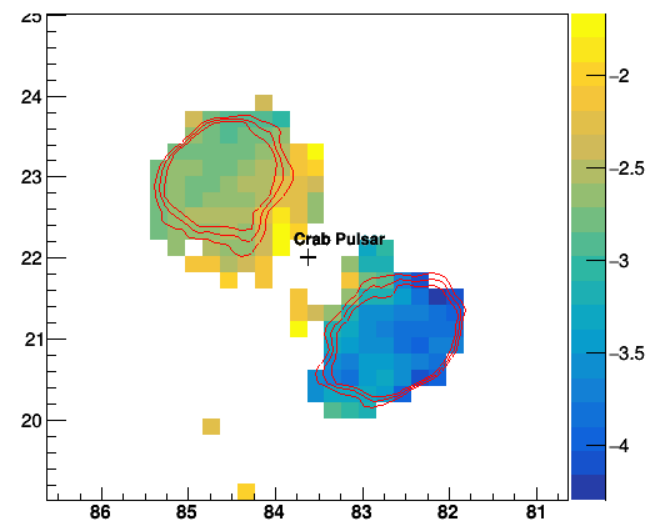

(b)

Figure 3: (a) Excess map and (b) spectral-index map of VERITAS signal-free data (Crab observation with the point source masked by a radius of $0.2^{\circ}$ ) injected with simulated $\gamma$-ray events of two extended sources $\left(r=0.5^{\circ}\right)$ with different spectral indices. The spectra are modeled as $F=N_{0}(E / \mathrm{TeV})^{-a}$. The top left source has an index of $a=2.5$, the bottom right source has an index of $a=3.5$.

morphology-resolved spectroscopic studies of VHE $\gamma$-ray sources with large angular extension such as SNRs, PWNe and their evolution. Given that MIBE makes no assumption about putative source position, it may become a valuable tool for the detection of transient sources with poor initial localization provided by the multi-messenger signals, such as gravitational waves and/or neutrinos. VHE $\gamma$-ray transient astrophysics may, therefore, benefit from MIBE application and further development.

\section{References}

[1] F. Aharonian et al. Observations of the Crab Nebula with H.E.S.S. Astron. Astrophys., 457:899915, 2006.

[2] David Berge, S. Funk, and J. Hinton. Background Modelling in Very-High-Energy gamma-ray Astronomy. Astron. Astrophys., 466:1219-1229, 2007.

[3] David J Fegan. $\gamma /$ hadron separation at TeV energies. Journal of Physics G: Nuclear and Particle Physics, 23(9):1013-1060, sep 1997.

[4] Giacinti, G., Mitchell, A. M. W., López-Coto, R., Joshi, V., Parsons, R. D., and Hinton, J. A. Halo fraction in tev-bright pulsar wind nebulae. A\&A, 636:A113, 2020.

[5] A. M. Hillas. Cerenkov Light Images of EAS Produced by Primary Gamma Rays and by Nuclei. In 19th International Cosmic Ray Conference (ICRC1985), volume 3, page 445-448, 1985. 
[6] Maria G. Krause, Elisa Pueschel, and Gernot Maier. Improved $\gamma /$ hadron separation for the detection of faint $\gamma$-ray sources using boosted decision trees. Astroparticle Physics, 89:1-9, 2017.

[7] Henric Krawczynski, D.A. Carter-Lewis, C. Duke, J. Holder, G. Maier, S. Le Bohec, and G. Sembroski. Gamma-Hadron Separation Methods for the VERITAS Array of Four Imaging Atmospheric Cherenkov Telescopes. Astropart. Phys., 25:380-390, 2006.

[8] Takahiro Sudoh, Tim Linden, and John F. Beacom. TeV halos are everywhere: Prospects for new discoveries. Physical Review D, 100(4):043016, August 2019. 